\title{
A New Low Complexity NLOS Identification Approach Based on Minimum Slope and Kurtosis of Energy Block for $60 \mathrm{GHz}$ System
}

\author{
Xiaolin Liang ${ }^{1}$, Hao Zhang ${ }^{1,}, 2$, Tingting $\mathrm{Lv}^{1}$, T. Aaron. Gulliver ${ }^{2}$, \\ ${ }^{1}$ College of Information Science and Engineering, Ocean University of China, \\ Qingdao, 266100, China; \\ ${ }^{2}$ Department of Electrical and Computer Engineering, University of Victoria, \\ Victoria V8W 3P6, Canada \\ xiaolin87liang@163.com,zhanghao@ouc.edu.cn, \\ lvtingting33@163.com,agullive@ece.uvic.ca
}

\begin{abstract}
NLOS channels are the major problem of indoor localization. In order to perform NLOS identification, in this paper, a novel NLOS identification technique is proposed which based on ratio values of minimum slope and kurtosis of energy block of the received signal using Energy Detector. IEEE 802.15.3c $60 \mathrm{GHz}$ channel models are used as examples and above statistics is found to be explained in detail. Simplicity of the approach lies in using of parameters of Energy-based time of arrival (TOA) estimation algorithm. The CM1 and CM2 channel models of IEEE 802.15.3c channel models are used. Numerical simulations results show that correct identification of channel models with the proposed approach is better than with multipath channel statistics based approach.
\end{abstract}

Keywords: NLOS identification, minimum slope, kurtosis, $60 \mathrm{GHz}$, Energy Detector, IEEE 802.15.3c

\section{Introduction}

The location of a mobile terminal (MT) can be estimated using different parameters of a received signal, such as time-of-arrival (TOA), angle-of-arrival (AOA), and/or the received signal strength (RSS). Impulse radio-60 GHz has a great potential for accurate ranging and localization systems due to its very wide bandwidth and capability in resolving individual multipath components [1-6]. Therefore, TOA of the received signal can be estimated with high accuracy for $60 \mathrm{GHz}$ systems if the first arriving path has been identified precisely. One of the major challenges for localization systems is mitigation of NLOS effects. If the direct path between a fixed terminal (FT) (An FT is usually a base station in a cellular network or an anchor node in a sensor network.) and MT is being obstructed, TOA of signal to FT will be delayed, which introduces a positive bias. Using such NLOS TOA estimates during the localization of MT position may significantly degrade positioning accuracy. Hence, FTs that are under NLOS condition have to be identified and their effects have to be mitigated.

NLOS identification and mitigation techniques have been discussed extensively in the literature, but mainly within cellular network framework [7-13]. For example, in [7], skewness of ranging measurements is compared with threshold for NLOS identification, where measurement noise variance is assumed to be known. In [8], a decision-theoretic NLOS identification framework is presented, where various hypothesis tests are discussed for known and unknown probability density functions (PDFs) of TOA measurements. 
Guvenc et al. $[9,13]$ used mean excess delay, root mean square (RMS) delay, and kurtosis of multipath channel as NLOS identification metrics. When statistics of kurtosis, mean excess, and RMS delays are priori known, likelihood ratio tests can be performed for hypothesis selection. Another method was proposed by Heidari et al. [10]. They tried to find the first detected path of the received signal as a peak of the filtered channel impulse radio. As similar to previous method, this technique also used joint likelihood function using mean excess delay $\tau_{\text {med }}$, total received power $P_{\text {tot }}$ and hybrid of power of the first detected path and TOA of the first detected path $\xi_{h y b}$. Venkatesh et al. [11] identified channel based on TOA, RSS, and RMS delay spread (RDS) of the received signal. The conditional distributions of TOA, RSS, and RDS estimates are functions of distance and channel state. Provided that physical distance between the transmitted and receive nodes is known exactly, the state of the channel can be identified by comparing the likelihood values for each of the estimates (TOA, RSS, and RDS), conditioned on the distance. Shimizu et al. [12] performed intensive measurements of path-loss and delay-profile characteristics of LOS and NLOS environments in a suburban residential area. Based on their analysis, they found that the delay spread was dependent on distance, and the NLOS delay spread was found to be several times larger than that of the LOS case. The skewness of delay spread for the NLOS cases ranged from 80 to $200 \mathrm{~ns}$, which was an order of magnitude larger than that of the LOS case.

In this paper, we propose a new LOS/NLOS identification approach for $60 \mathrm{GHz}$ signal, which is based on minimum slope and kurtosis of energy block of the received signal using energy detector(ED). Firstly, we use ED TOA Estimation algorithm for estimation TOA. Secondly, we characterize minimum slope and kurtosis of energy block of the received signal. Finally, we use a threshold test for LOS/NLOS identification.

The remainder of this paper is organized as follows. Section II describes signal and channel model. Section III describes LOS/NLOS identification approach and Section IV presents the results of the numerical simulations. The concluding remarks are given in section $\mathrm{V}$.

\section{System Model}

Currently, there are two important standards that have been developed for $60 \mathrm{GHz}$ wireless communications systems, IEEE 802.15.3c and IEEE 802.11ad [14-15]. In this paper, the channel models in IEEE 802.15. 3c standard are used because it is specifically designed for wireless personal area networks and thus encompasses typical indoor environments. Further, these are the most widely employed models for $60 \mathrm{GHz}$ systems. IEEE 802.15.3c standard was the first developed for high data rate short-range wireless systems. The physical layer was designed to support the transmission of data within a few meters at a maximum data rate of $2 \mathrm{Gbps}$. These models have been developed for communications in the frequency band 57 to $66 \mathrm{GHz}$ in indoor residential, indoor office and library environments (with differences largely due to LOS and NLOS characteristics) [16-20]. In this paper, a pulse position modulation time hopping $60 \mathrm{GHz}$ signal is employed for ranging purposes. The propagation delay $\hat{\tau}$, between the transmitter and receiver is estimated for use in localization.

\subsection{GHz Signal}

$60 \mathrm{GHz}$ signals have a very short duration (typically 100 picoseconds or less), and can be expressed as:

$s(t)=\sum_{-\infty}^{\infty} p\left(t-j T_{s}-C_{j} T_{c}-a_{j} \varepsilon\right)$

Where $T_{s}$ is symbol time. The Time Hopping code represented by $\mathrm{C}$ is a pseudorandom 
integer-valued sequence which is unique for each user to limit multiple access interference, and $T_{c}$ is the chip time. PPM time shift is $\varepsilon$ so that if $a_{j}$ is 1 , the signal is shifted in time by $\varepsilon$, while if $a_{j}$ is 0 , there is no shift. Many pulse shapes have been proposed for $60 \mathrm{GHz}$ systems. In this paper a Gaussian pulse is employed which is multiplied by the carrier signal to give:

$p(t)=\frac{\sqrt{2}}{\alpha} \exp \left(-2 \pi \frac{t^{2}}{\alpha^{2}}\right) \cos \left(2 \pi f_{c} t\right)$

Where $\alpha$ is shape factor and $f_{c}$ is carrier frequency which here is $f_{c}=60 \mathrm{GHz}$. A smaller shape factor results in a shorter duration pulse and a larger bandwidth.

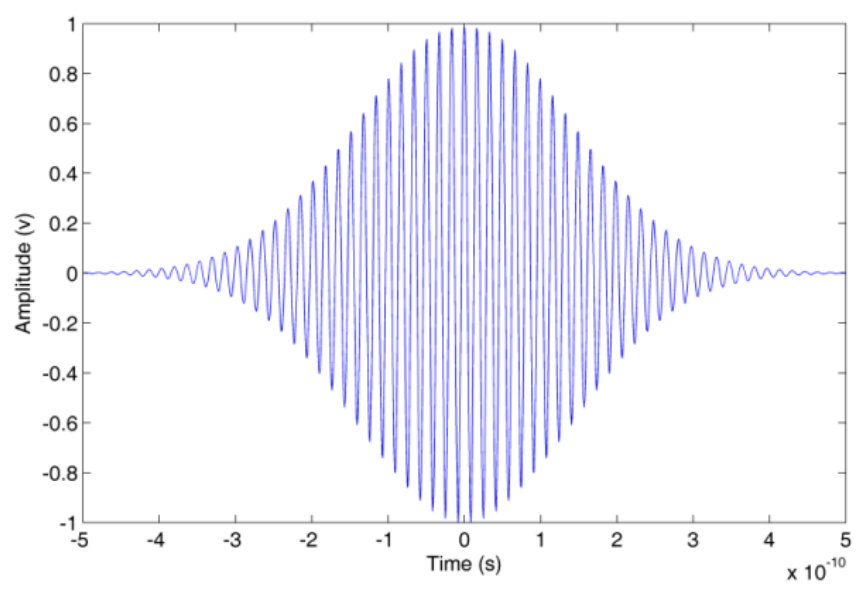

Figure 1 Waveform of $60 \mathrm{GHz}$ Signal

\subsection{Signal Shift and Path Loss}

The path loss is defined as the ratio of the received signal power to the transmit signal power and it is very important for link budget analysis. Unlike narrow-band system, path loss for a wide-band system such as mm-wave system, is both distance and frequency dependent. In order to simplify models, it is assumed that frequency dependence path loss is negligible and only distance dependence path loss is modeled. The signal path loss, which depends on the propagation distance and the channel (IEEE802.15.3c), is described by:

$P L(d)[d B]=P L_{0}+10 \cdot n \log _{10}\left(\frac{d}{d_{0}}\right)+X_{\sigma}[d B] ; d \geq d_{0}$

Where $d_{0}$ and $d$ denote reference distance, and distance respectively. $n$ is the path loss exponent $n$ for mm-wave, $X_{\sigma}$ is that the unit $\mathrm{dB}$, with mean zero and variance $\sigma_{s}$ for a Gaussian random variable. Table 1 summarizes values of $\mathrm{n}, P L_{0}, \sigma_{s}$ for different environments and scenarios.

Table1. Values of $\boldsymbol{n}, P L_{0}, \sigma_{s}$ for Different Environments and Scenarios

\begin{tabular}{cccc}
\hline environments & $n$ & $P L_{0}$ & $\sigma_{s}$ \\
\hline indoor residential (LOS) & 1.53 & 75.1 & 1.50 \\
indoor residential (NLOS) & 2.44 & 86.0 & 6.20 \\
\hline
\end{tabular}




\begin{tabular}{cccc}
\hline indoor office (LOS) & 1.16 & 84.6 & 5.40 \\
indoor office (NLOS) & 3.74 & 56.1 & 8.60 \\
\hline
\end{tabular}

The signal shift can be expressed as:

$t=d t *$ floor $((d / c) / d t)$

where $d$ denotes distance between the transmitter and receiver, $d t$ is sampling period and $c$ is speed of light which is $299792458 \mathrm{~m} / \mathrm{s}$ in the air.

\subsection{Multipath Fading Channel}

The received signal can be written as:

$r(t)=\sum_{n=1}^{N} \alpha_{n} p\left(t-\tau_{n}\right)+n(t)$

where $N$ is number of received multipath components, $\alpha_{n}$ and $\tau_{n}$ denotes amplitude and delay of the $n^{\text {th }}$ path respectively, $p(t)$ is the received $60 \mathrm{GHz}$ pulse and $n(t)$ is additive white Gaussian noise with zero mean and two sided power spectral density $\mathrm{N}_{0} / 2$. Equation (5) can be rewritten as:

$r(t)=s(t) * h(t)+n(t)$

where $s(t)$ is the transmitted signal, and $h(t)$ is the channel impulse response which can be expressed as:

$h(t, \theta)=\sum_{k=1}^{K} \sum_{l=1}^{L_{k}} \mu_{k l} \delta\left(t-T_{k}-\tau_{k l}\right) \delta\left(\theta-\theta_{k}-\omega_{k l}\right)$

Where $\delta($.$) is dirac-delta function, K$ is number of clusters, $L_{k}$ is number of rays in the $k^{\text {th }}$ cluster, and $\mu_{k l}, \tau_{k l}$ and $\omega_{k l}$ denote complex amplitude, delay and azimuth of the $k^{\text {th }}$ ray of the $l^{\text {th }}$ cluster, respectively. Similarly, $T_{k}$ and $\theta_{k}$ represent delay and mean AOA of the $k^{\text {th }}$ cluster.

\subsection{Energy Detector}

After the amplifier, the received signals are squared, and then input to an integrator with integration period $T b$. Because of inter-frame leakage due to multipath signals, integration duration is $3 T_{f} / 2$, so number of signal values for $\mathrm{ED}$ is $\mathrm{N}=3 T_{f} / 2 T b$. The integrator outputs can be expressed as:

$$
z[n]=\sum_{i=1}^{N} \int_{(i-1) T_{f}+\left(c_{j}+n-1\right) T b}^{(i-1) T_{f}+\left(c_{j}+n\right) T b} r^{2}(t) d t
$$

Where $n \in\{1,2, . ., N\}$ denotes sample index with respect to starting point of integration period and $N$ is number of pulses per symbol. Here, $\mathrm{N}$ is set to 1 , so integrator outputs are:

$z[n]=\sum_{i=1}^{N} \int_{\left(c_{j}+n-1\right) T b}^{\left(c_{j}+n\right) T b} r^{2}(t) d t$

If $z[n]$ is the integration of noise only, it has a centralized Chi-square distribution, while it has a non-centralized Chi-square distribution if a signal is present. The mean and variance 
of the noise and signal values are given by [17] respectively.

$$
\begin{aligned}
& \mu_{0}=F \sigma^{2}, \sigma_{0}=2 F \sigma^{4} \\
& \mu_{e}=F \sigma^{2}+E_{n}, \sigma_{e}^{2}=2 F \sigma^{4}+4 \sigma^{2} E_{n}
\end{aligned}
$$

Where $E_{n}$ is signal energy within the $n^{\text {th }}$ integration period and $F$ is number of degrees of freedom given by $F=2 B T b+1$. Here $B$ is signal bandwidth.

\section{LOS/NLOS Identification}

\subsection{Description of Parameters}

In this paper, we distinguish between LOS or NLOS scenarios by exploiting statistics of the received signal by ED. The minimum slope and kurtosis of energy block is used in order to identify LOS and NLOS scenarios respectively. The slope of the energy values is considered as a measure. These values are divided into $(\mathrm{N}-\mathrm{M}+1)$ groups, with $\mathrm{M}$ values in each group. The slope for each group is calculated using a least squares line-fit. The minimum slope can then be expressed as:

$M S=\min _{1 \leq n \leq N-M+1}$ slope $\{$ linefit $(z[n], z[n+1], \ldots . ., z[n+M-1])\}$

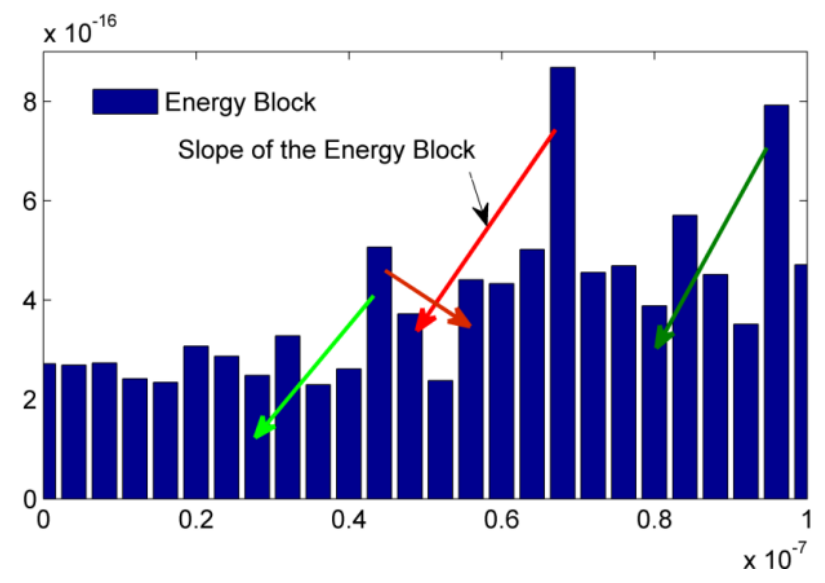

Figure 2. Slope of Energy Block

Kurtosis is calculated using the second and fourth order moments and is given by:

$$
K=\frac{E\left[\left(x_{i}-\mu_{x}\right)^{4}\right]}{E\left[\left(x_{i}-\mu_{x}\right)^{2}\right]^{2}}=\frac{E\left[\left(x_{i}-\mu_{x}\right)^{4}\right]}{\sigma_{x}^{4}}
$$

where $\mu_{x}$ is mean value, and $\sigma_{x}$ is standard deviation. Kurtosis for a standard normal distribution is three. For this reason, Kurtosis is often redefined as $K=K-3$ (often referred to as "excess Kurtosis"), so that the standard normal distribution has a Kurtosis of zero, positive Kurtosis indicates a "peaked" distribution and negative Kurtosis indicates a "flat" distribution. For noise only (or for a low SNR) and sufficiently large $F$ (degrees of freedom of the Chi-square distribution), $z[n]$ has a Gaussian distribution and Kurtosis $=0$. On the other hand, as the SNR increases, Kurtosis will tend to increase.

\subsection{Identification Approach}


The ratio values of slope and kurtosis of energy block can be obtained for both LOS and NLOS scenarios using sample channel realizations from both scenarios. Here, we used sample channel realizations of IEEE 802.15.3c standard channel models in order to obtain the values of slope of energy slope for both LOS and NLOS. In order to examine the characteristics of ratio of minimum slope and kurtosis, CM1.1 and CM2.1 from IEEE 802.15.3c standard are employed. For each SNR value, 1000 channel realizations are generated and sampled at $f_{c}=1 \cdot e^{10} \mathrm{~Hz}$. The other system parameters are $T_{f}=200 \mathrm{~ns}, T_{c}=1 \mathrm{~ns}$, the value of $T b$ is from $1 \mathrm{~ns}$ to $4 \mathrm{~ns}$ and $N=1$. Each realization has a TOA uniformly distributed within $\left(0-T_{f}\right)$. Here SNR ranges from $0 \mathrm{~dB}$ to $7 \mathrm{~dB}, 1000$ channel realizations are generated for each SNR. CM1.1 and CM2.1 from IEEE 802.15.3c standard are employed. Here are $8 * 1000$ samples which are got for each channel model. The relationship between ratios and SNR are shown in Figures 3-6.

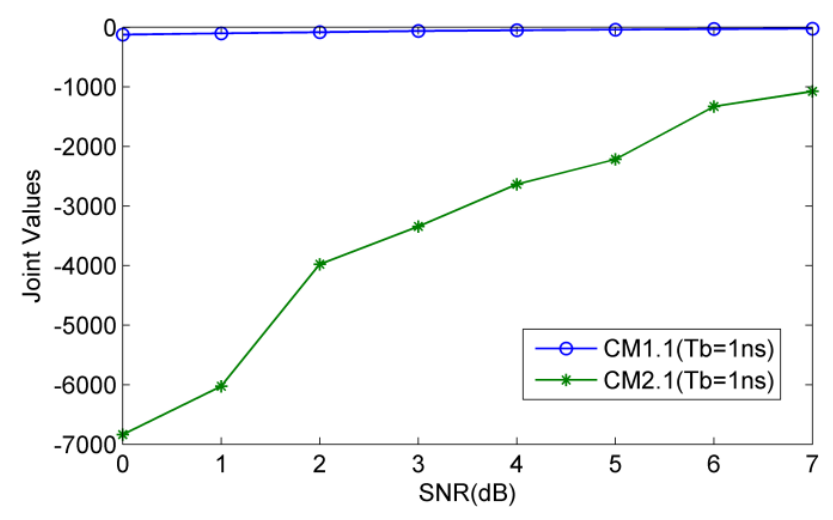

Figure 3. Ratios with Respect to SNR (CM1.1 and CM2.1 with Tb=1ns)

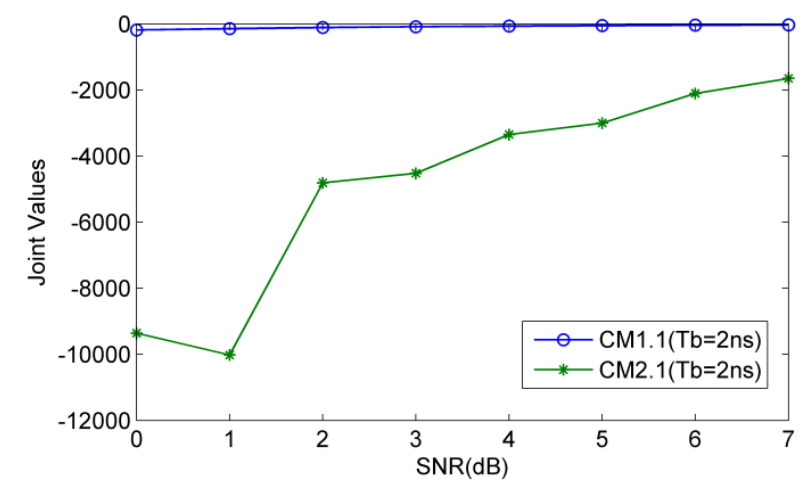

Figure 4. Ratios with Respect to SNR (CM1.1 and CM2.1 with Tb=2ns) 


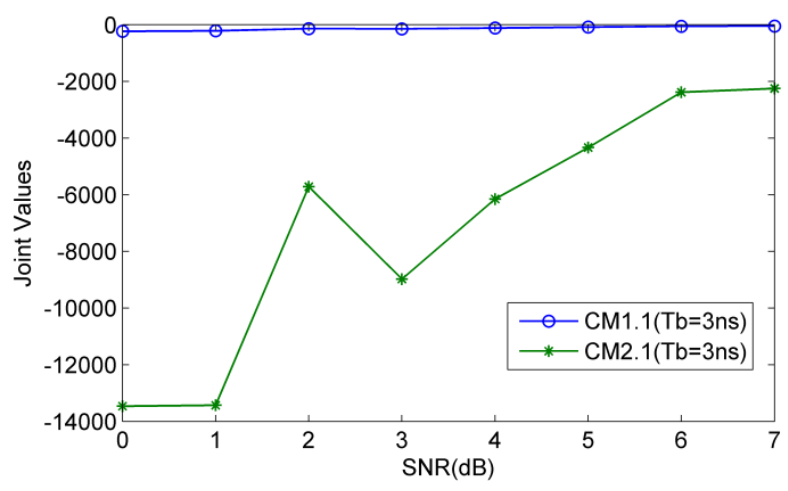

Figure 5. Ratios with Respect to SNR (CM1.1 and CM2.1 with Tb=3ns)

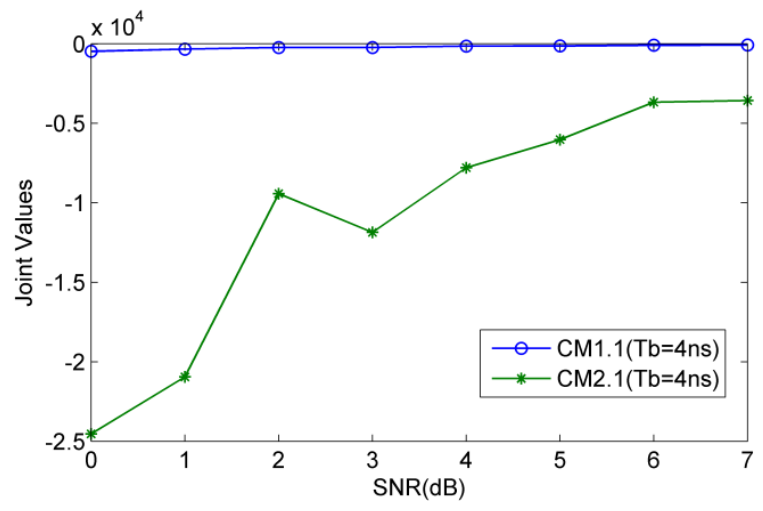

Figure 6. Ratios with Respect to SNR (CM1.1 and CM2.1 with Tb=4ns)

From Figures 3-6, results show that ratio values are monotonous with respect to SNR (both LOS and NLOS environment) in $60 \mathrm{GHz}$ wireless communication system respectively. But we find minimum of joint values (LOS) is even larger than maximum of joint values (NLOS) when TX is 360, maximum of joint values (LOS) is even less than minimum of joint values (NLOS) when TX is others. So we propose a novel method to identify the LOS and NLOS which can be expressed as:

$\Theta=\alpha\left\{\begin{array}{l}\left\{\begin{array}{l}<\alpha_{M S} \Rightarrow N L O S \\ >\alpha_{M S} \Rightarrow L O S\end{array} \quad T X=360^{\circ}\right. \\ \left\{\begin{array}{l}<\alpha_{M S} \Rightarrow L O S \\ >\alpha_{M S} \Rightarrow N L O S\end{array} \quad \text { others }\right.\end{array}\right.$

where $\alpha_{M S}$ is threshold which is chosen to identify LOS/NLOS. TX, RX is the beam-width of measured transmitter and receiver antenna respectively. Aiming at the ranges $[\mathrm{A}, \mathrm{B}] \mathrm{dB}$ for SNR, $\alpha_{M S}$ can be shown as follows :

$\alpha_{M S}=\max \left\{\begin{array}{l}\operatorname{mean}\left[\operatorname{find}\left(P\left(M S>\left.\Theta_{1}\right|_{A}\right) \geq \Phi\right)\right] \\ \operatorname{mean}\left[\operatorname{find}\left(P\left(M S>\left.\Theta_{2}\right|_{B}\right) \geq \Phi\right)\right]\end{array}\right\}$

where $\Theta_{1}$ and $\Theta_{2}$ are threshold which meet the condition $P(M S>\Theta) \geq \Phi$ where SNR is upper and lower limits. $\Phi$ is the probability value which is required for choosing suitable threshold for NLOS identification. As shown in Figure 7, the distribution of K/M samples 
larger than a certain threshold value $\Theta$ for the IEEE802.15.3c channel. When $\Theta$ is set to be 1 and more than 950 of the $1000 \mathrm{~K} / \mathrm{M}$ samples are distributed less than $\Theta$. In the process of simulation, results show that if we use those samples whose probability distribution is high, we can guarantee accuracy of NLOS identification and remove some extreme values at the same time. Without loss of generality, here $\Phi$ is set to be 0.85 .

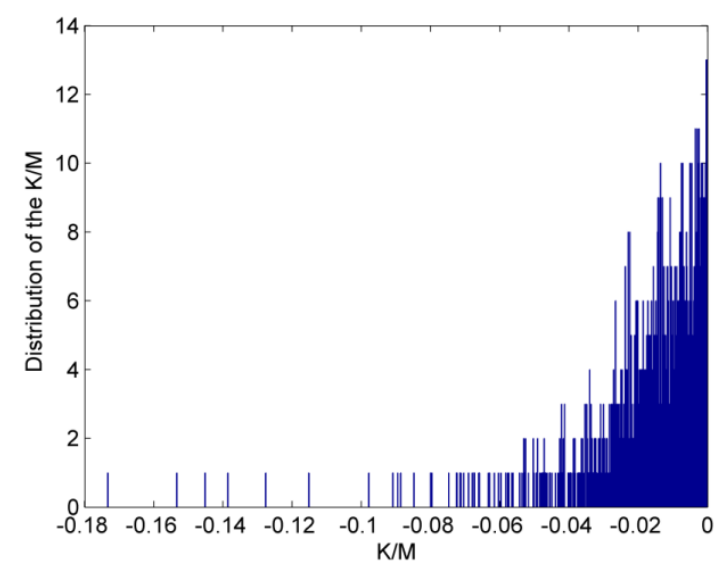

Figure 7. Distribution of Ratios

\section{Simulated Results}

In order to verify the effectiveness and practicality of the algorithm, so we make a lot of simulations using IEEE 802.15.3c channel models. The CM1.2, CM1.3, CM1.4 (residential LOS) and CM2.2, CM2.3, CM2.4 (residential NLOS) channel models from the IEEE802.15.3c standard are employed. For each SNR value, 1000 channel realizations are generated and sampled at $f_{c}=1 \cdot e^{10} \mathrm{~Hz}$. The other system parameters are $T_{f}=200 \mathrm{~ns}$, $T_{c}=1 n s$, the value of $T b$ is from $1 \mathrm{~ns}$ to $4 \mathrm{~ns}$ and $\mathrm{N}=1$. Each realization has a TOA uniformly distributed within $\left(0-T_{f}\right)$. Here the SNR ranges from $0 \mathrm{~dB}$ to $7 \mathrm{~dB}, 1000$ channel realizations are generated for each SNR. The CM1.2, CM1.3, CM1.4 (residential LOS) and CM2.2, CM2.3, CM2.4 (residential NLOS) channel models from the IEEE802.15.3c standard are employed. Here are $8 * 1000$ samples which are got for each channel model. The relationship between joint parameter and SNR are shown in Figures 8-19.

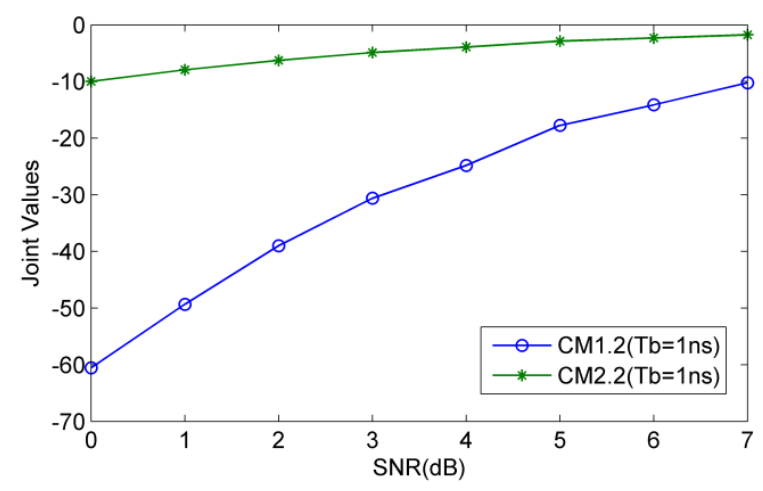

Figure 8. Ratios with Respect to SNR (CM1.2 and CM2.2 with $\mathrm{Tb}=1 \mathrm{~ns}$ ) 


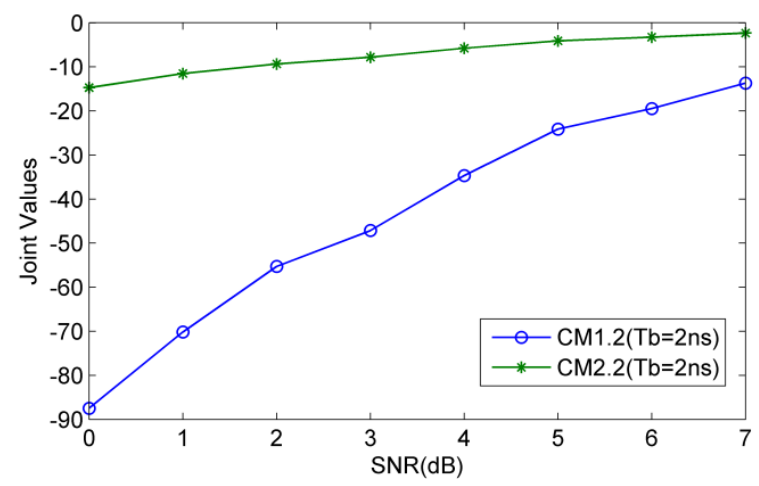

Figure 9. Ratios with Respect to SNR (CM1.2 and CM2.2 with Tb=2ns)

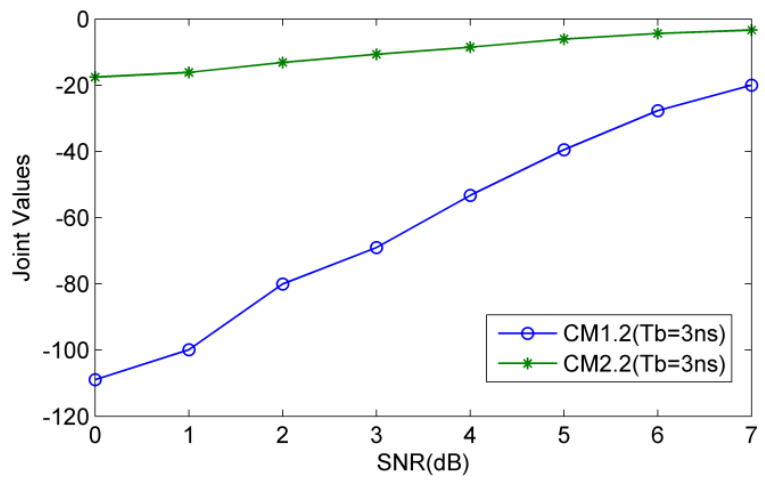

Figure 10. Ratios with Respect to SNR (CM1.2 and CM2.2 with Tb=3ns)

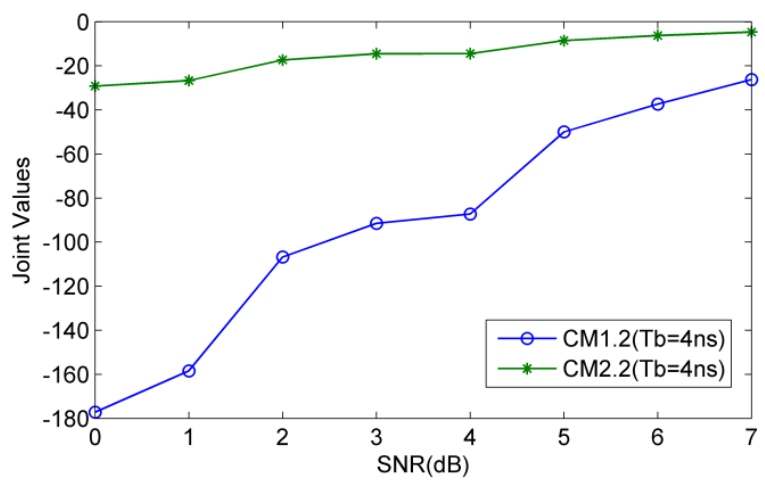

Figure 11. Ratios with Respect to SNR (CM1.2 and CM2.2 with Tb=4ns) 


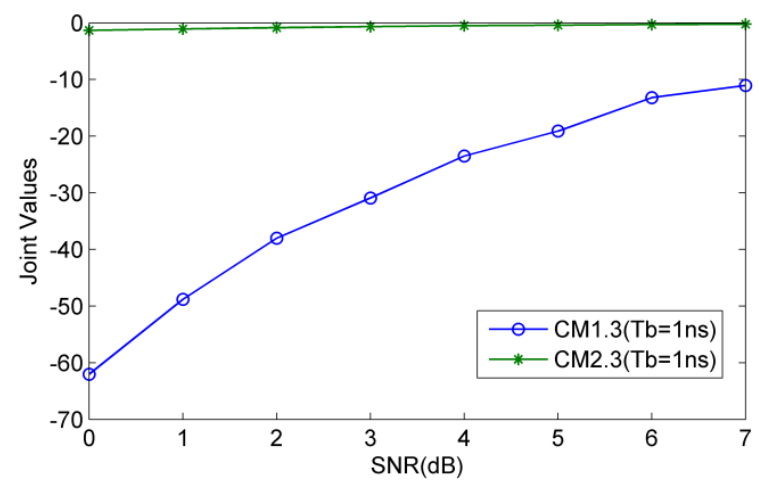

Figure 12. Ratios with Respect to SNR (CM1.3 and CM2.3 with Tb=1ns)

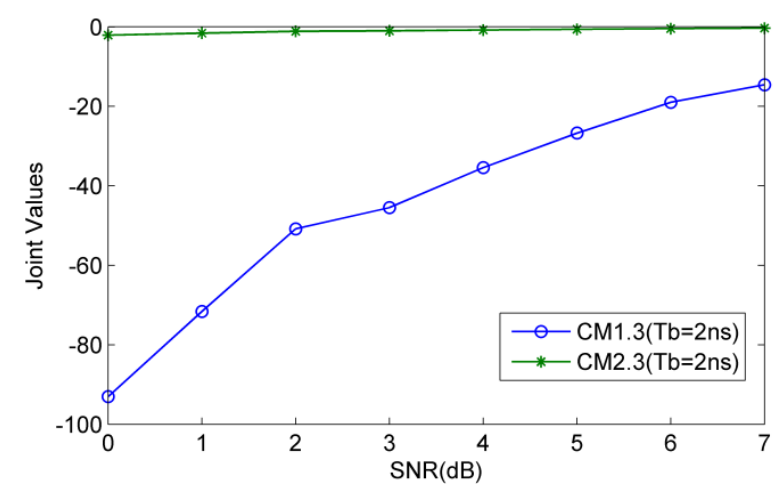

Figure 13. Ratios with Respect to SNR (CM1.3 and CM2.3 with Tb=2ns)

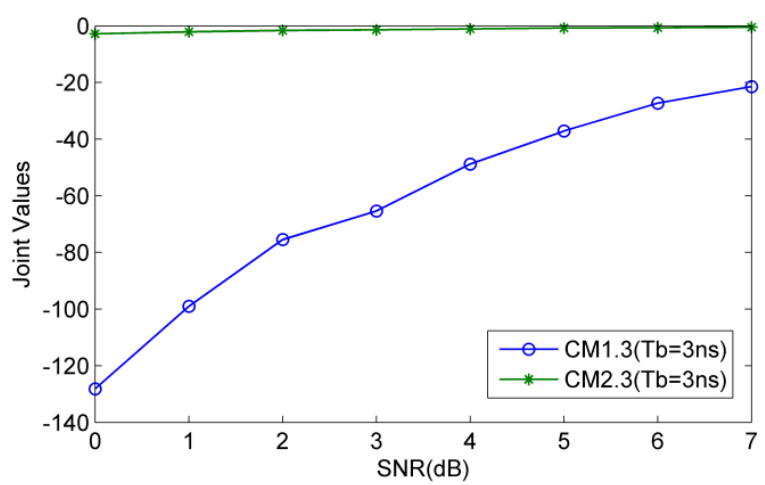

Figure 14. Ratios with Respect to SNR (CM1.3 and CM2.3 with Tb=3ns) 


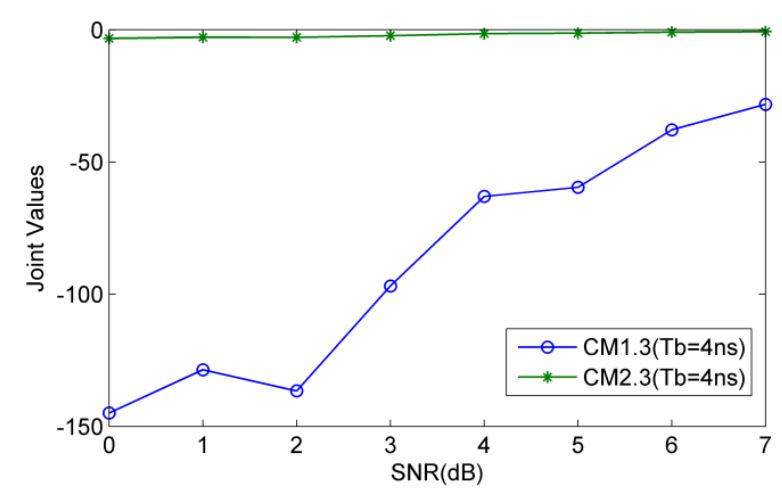

Figure 15. Ratios with Respect to SNR (CM1.3 and CM2.3 with Tb=4ns)

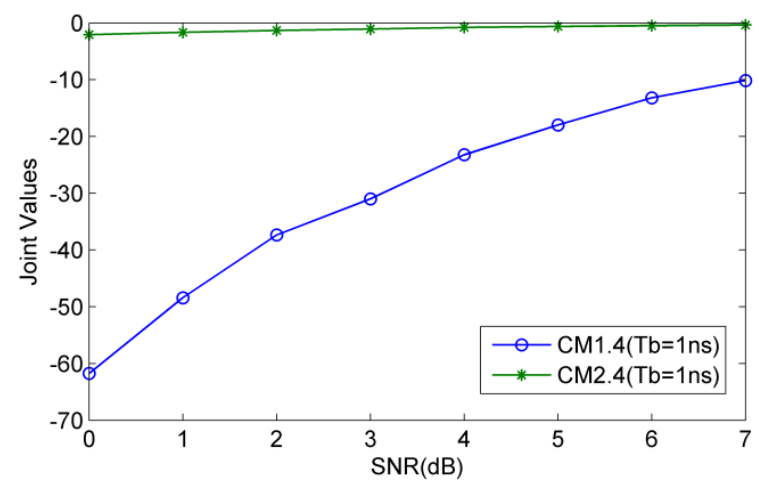

Figure 16. Ratios with Respect to SNR (CM1.4 and CM2.4 with Tb=1ns)

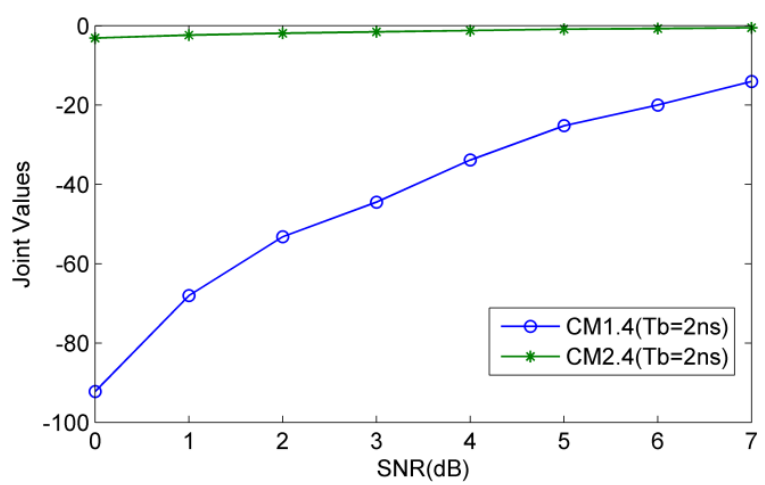

Figure 17. Ratios with Respect to SNR (CM1.4 and CM2.4 with Tb=2ns) 


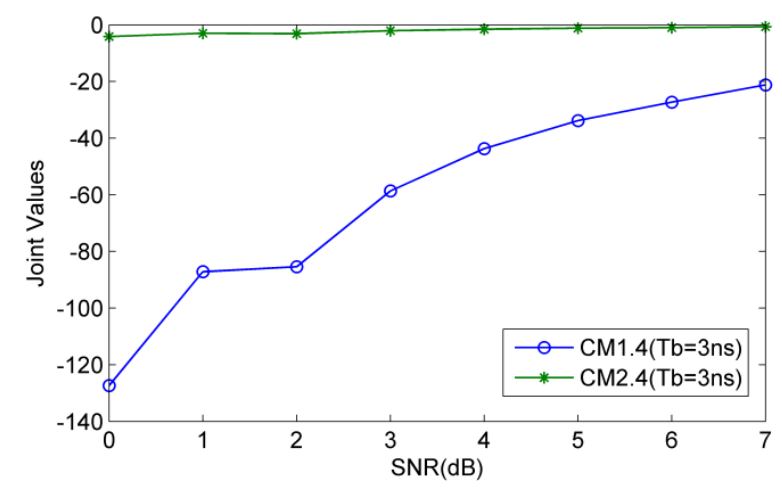

Figure 18. Ratios with Respect to SNR (CM1.4 and CM2.4 with Tb=3ns)

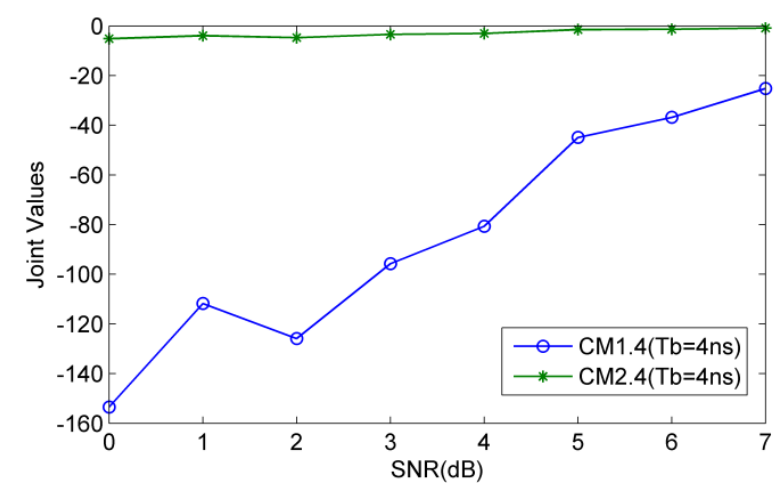

Figure 19. Ratios with Respect to SNR (CM1.4 and CM2.4 with Tb=4ns)

From Figures 8-19, results show that joint parameter can identify LOS and NLOS environments so long as threshold $\alpha_{M S}$ can be fixed better.

\section{Conclusion}

In this paper, we presented a novel approach to deal with non-line-of-sight propagation that relies solely on features extracted from received waveform. This technique does not require formulation of explicit statistical models for the features which is based on minimum slope and kurtosis of energy block of the received signal using ED.

In order to verify effectiveness and practicality of the algorithm, so we make a lot of simulations using IEEE 802.15.3c channel models. The CM1.1, CM1.2, CM1.3, CM1.4 (residential LOS) and CM2.1, CM2.2, CM2.3, CM2.4 (residential NLOS) channel models from the IEEE802.15.3c standard are employed. Results show that the joint parameter can identify the LOS and NLOS environments so long as the threshold $\alpha_{M S}$ can be fixed bitterly.

We developed techniques that are capable of distinguishing LOS/NLOS propagation in NLOS conditions. Our results revealed that the proposed technique outperforms previous parametric techniques from the literature. But here is a question that the method we proposed can't identify the LOS and NLOS environments in the office. So in the future, this will be the problem which is eager to be solved for us 


\section{Acknowledgments}

The authors would like to thank colleagues from UWB Laboratory in College of Information Science and Engineering, Ocean University of China, for help with obtaining measurement data. This work was supported by Nature Science Foundation of China under Grant No. 60902005, Qingdao International Science and Technology Cooperation Projects of Qingdao under Grant No. 12-1-4-137-hz, and Qingdao Transformation of Scientific and Technological Achievements Guiding Plan (youth special program) under Grant No. 14-2-4-37-jch.

\section{Reference}

[1] L. Zhang, "A fully integrated $60 \mathrm{GHz}$ four channel CMOS receiver with $7 \mathrm{GHz}$ ultra-wide band width for IEEE 802.11ad standard", Communication, China, vol. 11, no. 6, (2014), pp. 42-50.

[2] S. K. Yong and C. C. Chong, "An overview of multi gigabit wireless through millimeter Wave Strategy: Potentials and Technical Challenges", EURASIP J. Wireless Communications and Networking, vol. 2007, no. 1, (2007), pp. 1-10

[3] R. C. Daniels and R. W. Heath, "60 GHz wireless communications: emerging requirements and design recommendations", IEEE Vehicular Strategy Society, vol. 2, (2007), pp. 41-50.

[4] C. C. Chong, F. M. Peter, Smulders, et al, "60GHz-Millimeter-Wave Radio Principle, Strategy, and News Results", EURASIP Journal on Wireless Communications and Networking, vol. 2007, no. 1, (2007), pp. 1-8.

[5] S. K. Yong, P. F. Xia P F and Alberto V G, "60-GHz Strategy for Gbps WLAN and WPAN: From Theory to Practice", Beijing: Press of China Machine, (2013).

[6] R. C. Daniels and R. W. Health, "60 GHz wireless communications: emerging requirements and design recommendations", IEEE Vehicular Strategy Magazine, vol. 2, no. 3, (2007), pp. 41-50.

[7] M. P. Wylie and J. Holtzman, "The non-line of sight problem in mobile location estimation," in Proceedings of the 5th IEEE International Conference on Universal Personal Communications (ICUPC '96), Cambridge, Mass,USA, vol. 2, September-October (1996), pp. 827-831.

[8] J. Borras, P. Hatrack, and N. B. Mandayam, "Decision theoretic framework for NLOS identification," in Proceedings of the 48th IEEE Vehicular Technology Conference (VTC '98), Ottawa, Canada, vol. 2, May (1998), pp. 1583-1587.

[9] Guvenc I, Chong C-C, Watanabe F (2007) NLOS identification and mitigation for UWB localization systems. Wireless Communications and Networking Conference. WCNC 2007. IEEE, (2007), pp. 15711576.

[10] M. Heidari, FO Akgul, K Pahlavan, "Identification of the absence of direct path in indoor localization systems", IEEE International Symposium on Personal, Indoor, and Mobile Radio Communications, (2007), pp. 1-6.

[11] S. Venkatesh, RM Buehrer, (2007) Non-line-of-sight identification in ultra-wideband systems based on received signal statistic. Microwaves, Antennas \& Propagation, IET, vol. 1, no. 6, pp. 1120-1130.

[12] H. Shimizu, H. Masui, M. Ishii, K. Sakawa, (2000) LOS and NLOS path-loss and delay characteristics at $3.35 \mathrm{GHz}$ in a residential environment. Antennas and Propagation Society International Symposium, 2000. IEEE, 2:1142-1145

[13] I. Güvenç, C-C Chong, F. Watanabe, H. Inamura, (2008) NLOS identification and weighted least-squares localization for UWB systems using multipath channel statistics. EURASIP Journal on Advances in Signal Processing 2008:14 Article ID 271984

[14] "IEEE Standard for Information strategy--Local and metropolitan area networks--Specific requirements--Part 15.3: Wireless Medium Access Control (MAC) and Physical Layer (PHY) Specifications for High Rate Wireless Personal Area Networks (WPAN) amendment 2: millimeter-wave-based alternative physical layer extension". IEEE Computer Society, IEEE 802.15.06-0474-00-003c. New York, USA, (2009).

[15] "802.11n-2009-IEEE Standard for Information strategy-- Local and metropolitan area networks--Specific requirements--Part 11: Wireless LAN Medium Access Control (MAC)and Physical Layer (PHY) Specifications Amendment 5: Enhancements for Higher Throughput", IEEE Computer Society, IEEE 978-0-7381-6731-2. New York, USA, (2009).

[16] C. R. Andersonn and T. S. Rappaport, "In-building wideband partition loss measurements at 2.5 and 60GHz", IEEE Transactions on Wireless Communications, vol. 3, no. 3, (2004), pp. 922-928.

[17] S. Collong, G. Zaharia and G. E. Zein, "Influence of the human activity on wide-band characteristics of the $60 \mathrm{GHz}$ indoor radio channel", IEEE Transactions on Wireless Communications, vol. 3, no. 6, (2005), pp. 2396-2406. 
[18] A. Maltsev, R. Maslennikov and A. Sevastyanov, "Experimental investigations of 60GHz WLAN systems in office environment", IEEE Journal on Selected Areas in Communications, vol. 27, no. 8, (2009), pp. 1488-1499.

[19] M. G. Sanchez, A. V. Alejos and I. Cuinas, "Comparision of space diversity performance in indoor radio channels at $40 \mathrm{GHz}$ and $60 \mathrm{GHz}$ ", Proc. of European Conference on Wireless Strategy, Amsterdam, (2008).

[20] H. B. Yang, "Channel characteristics and transmission performance for various channel configurations at 60GHz", EURASIP Journal on Wireless Communications and Networking, vol. 2007, no. 1, (2007), pp. 43-43.

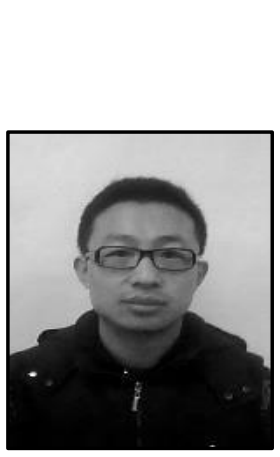

\begin{abstract}
Authors
Xiaolin Liang, He now studies in College of Information Science and Engineering and is a $\mathrm{Ph}$. D. candidate in Ocean University of China. His research interests include ultra-wideband radio systems, $60 \mathrm{GHz}$ wireless communication system.
\end{abstract}

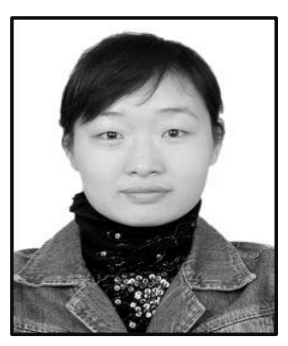

Tingting Lv, She received $\mathrm{Ph}$. D. degree in College of Information Science and Engineering from Ocean University of China in 2013. She is now a lecture in College of Information Science and Engineering. Her research interests include ultra-wideband radio systems, $60 \mathrm{GHz}$ wireless communication system.

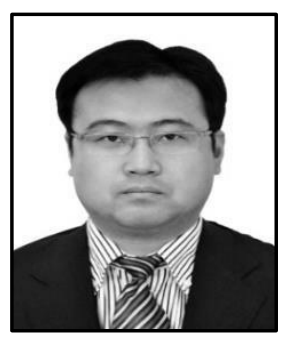

Hao Zhang received MBA degree in New York Institute of Technology, American in 2001 and Ph. D. degree in Electrical and Computer Engineering from University of Victoria, Canada in 2004. He was a Project Manager for Microsoft Inc. in United States during January 2000-May 2000. During 2004-2008, he was Vice President for the United States Gamma Capital Inc. He is now an Adjunct Assistant Professor in Department of Electrical and Computer Engineering. Also he becomes a professor and Ph. D. supervisor in College of Information Science and Engineering from Ocean University of China in 2006. His research concerns ultra-wideband radio systems, $60 \mathrm{GHz}$ wireless communication system and MIMO wireless communication.

T. Aaron. Gulliver received $\mathrm{Ph}$. D. degree in Electrical and Computer Engineering from the University of Victoria, Canada in 1989. He is now a professor and Ph. D. supervisor in Department of Electrical and Computer Engineering. In 2002, he becomes a Fellow of the Engineering Institute of Canada, and in 2012 a Fellow of the Canadian Academy of Engineering. He is also a senior member of IEEE. His research concerns information theory and communication theory, algebraic coding theory and smart grid and ultra-wideband communication. 